\title{
Language and Human-Computer Interaction: Meeting the Needs of a Rapidly Changing World
}

\author{
Dr. A. Belmekki \\ Senior Lecturer, Abu Bakr Belkaid University ,Tlemcen \\ Faculty of Letters and Languages, \\ Department of Foreign Languages, Section of English \\ E-mail : ambel1971@yahoo.co.uk, Fax: 043.21.15.32
}

\section{Doi:10.5901/mjss.2013.v4n3p273}

\begin{abstract}
As the developed world moves from the industrial age to the information age, little in this world is changing as fast as language education and educational technology. It is challenging to believe that computers are supposed to provide individualized instruction in almost every subject, and which are valuable tools for building basic skills, developing problem-solving strategies and, thus, providing innovative methods for languages. Henceforth, the present paper calls for ways of how may computers affect individuals, managing, at the same time, computer use in language learning by establishing a sound interaction between computers and human minds.Computer networks offer language teachers and students reliable help by sharing information and other resources as schools develop new ways of applying technology. In a word, human language technologies (HLT) hold much promise for the developing world.
\end{abstract}

Keywords: Languages and Human-Computer Interaction, human language technologies (HLT).

With the rapid changes in computer technology, debates are sought to find out in what ways computers can be used to enhance language teaching/learning. Human-Computer Interaction, for short $\mathrm{HCl}$, is a discipline mainly concerned with the design, evaluation and implementation of interactive computing systems for human use and with the study of major phenomena surrounding them. Computer systems are unique among artificial devices in allowing for a substantive, intelligent interaction between human beings and artifacts. By contrasting the two domains, namely computer and language learning to find out where usefulness exist and where it does not, one may obtain insights into the nature of computer system design and a richer perspective on the constraint on learning languages.

Research on the effectiveness of new technologies in education, including the use of computers in language teaching, has been an ongoing process. This has led many pedagogical specialists to consider possible combinations and interactions between language learning and computer use. For instance, a graduate student who set out to show the effectiveness of books in the classroom would quickly be counselled to refine the topic in order to make it meaningful, and the same advice would be given to someone trying to measure computer effectiveness in the classroom. Chapelle and Jamieson (1990) have long suggested that research on computers incorporate the areas known to be relevant in language acquisition, such as learner field dependence/independence and learning strategies.

Foreign language teachers have always been ahead of the curve in integrating technology in FL instruction and learning, seeing the benefits of technology even without an extant research database to confirm their judgment. In this respect, Lee (2000) states the necessity of the application of computer technology in language instruction in the following adapted points:

* Raising the learners' motivation,

* Enhancing students' achievement and progress,

* Increasing authentic materials and activities for study,

*ncouraging greater interaction between teachers/ students, and students/ peers,

* Emphasizing the individual needs,

* Reducing the learning stress and anxiety, thus increasing their self-confidence,

* Providing repeated lessons as often as necessary.

* Helping learners strengthen their linguistic skills, affect their learning attitude, and build their self-instruction strategies. 
As computers get faster and more reliable, language learning skills benefit from computer use for, it offers successful results in a small period of time. Writing is perhaps the skill most investigated by Second Language Acquisition (SLA) researchers; it seems that language learners demonstrate increased target language production when using writing assistants. This increased production is sometimes judged to be qualitatively better than that produced without the use of computer assistance, but the results are not unanimous in this regard. (Gray \& Stockwell, 1998; Hyland, 1993; Kern, 1995; Liu et al., 2002; Singhal, 1998; Warschauer \& Healey, 1998).

It is worth pointing that within literature, it appears very few studies concentrating specifically on the skill of listening. Clearly one may benefit from computers in this area for the increased access to target language input presented in a variety of ways. For instance, the multimedia capabilities of computers enable learners engage in a complex listening experience, complete with visual cues from the interlocutor. The greatest advantage touted in research on listening and computer is that the multimedia nature of the activities addresses the use of different modalities, thus appealing to a wider variety of learning styles. (Liu et al., 2002).

In the early days, with the large mainframe computers, the task of creating language software programmes was rather daunting, as developers needed to have a deeper knowledge of programming languages. In more recent times, though, computers have become more "user-friendly" and, therefore, easier even for non-specialists such as language teachers to use, it is enabling them to create more complex applications with relative ease. As far as speaking is concerned, a great number of speech recognition soft wares has been explored as a possible aid to language learning. The general consensus is that, while these soft wares show promise for future research, they are not yet sufficiently developed or reliable to justify its use in foreign language (FL) studies. (Liu et al., 2002). Pronunciation as a skill also benefited from computers, Automatic Speech Recognition (ASR) is in fact, a pronunciation training programme in which learners read sentences on the screen and the computer gives feedback as to the accuracy of the utterance, usually in the form of visual sound waves.

As for studies on the use of computer to improve reading skills, the primary stress has been placed on the use of glosses and vocabulary acquisition. Students make use of computer technologies to assist their reading comprehension. In addition to this, they may identify unknown vocabulary using computers' provided synonyms to ease text comprehension.

At another layer of analysis, it is of crucial importance to note that language learning may be impeded by anxiety and lack of motivation which act as filters blocking comprehension (Krashen, 1989), technology, however, can assist teachers in creating a supportive affective environment in the classroom.

Seeing that equipped computer laboratories may handle probably all requirements of language laboratories gave advantages of the language laboratory, which can be said for computer laboratories, too. Huebener (1960:112):

* All objectives of a language course can be achieved in the laboratory: speaking, understanding, pronouncing, learning vocabulary, carrying on a conversation, memorizing, reading and dictation.

* The near-ideal pronunciation of native speakers is always available in the laboratory.

* The student becomes readily accustomed to different kinds of voices. In the conventional classroom he hears only his teacher's voice.

* In the laboratory the student can listen over and over again.

* The laboratory gives every student an opportunity to practice individually during the whole period.

* By constant repetition and oral drill the student acquires with ease a new set of speech habits- the real objective of learning a foreign language.

Although there are many advantages of computer application in language instruction, computer technology still has its limitations and disadvantages. For instance, computers cannot handle unexpected situations, as learning situations are various and ever changing and influenced by a chemistry of variables, it is pretty impossible for the computer to be ready to deal with the learners' unexpected learning problems and response to learners' questions immediately as teachers do.

It is clear that all of the most vital innovations in language education have greatly benefited from computer development. It can be drawn from this article that the role of computers in language teaching has changed significantly in the last 30 years. Previously, computers were used principally for drills and exercises. Technological and pedagogical developments now allow us to better integrate computer technology into the language learning process. Multimedia programmes incorporating speech-recognition software may in all probabilities immerse students into rich environments for language practice. Concordancing software and large language corpora provide students the means to investigate language use in authentic contexts. And the Internet allows for a myriad of opportunities to communicate in the target language, access textual and multimedia information, and publish for a global audience. 
Future developments in networked communication, multimedia, and artificial intelligence is thought to likely converge, creating a potentially more central role for the computer as a tool for authentic language exploration and use in a foreign language context. As our focus of attention gradually shifts from the computer itself to the natural integration of computers into the language learning process, it will be acknowledged that computer technology is supposed to occupy its rightful place as a significant element of language learning and teaching.

To conclude, as an emergent layer we can characterize and interpret $\mathrm{HCl}$ via the communicative interaction cycle between user, interface and system in terms of design, interaction and process patterns. This is a new terminological framework for $\mathrm{HCl}$ and especially for research on usability. The following diagram illustrates this clearly.

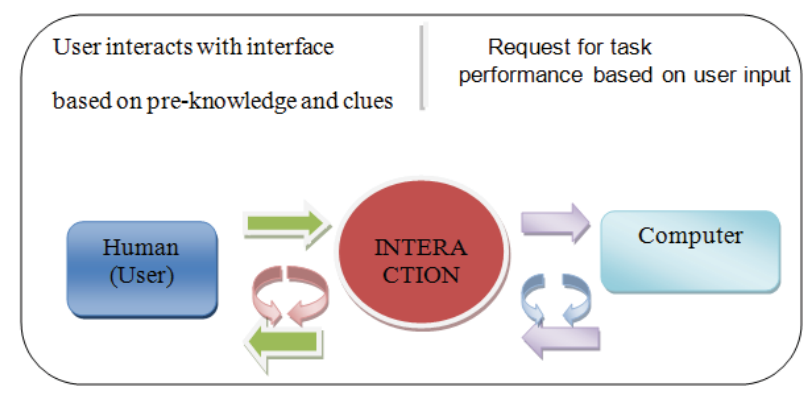

Diagram 1. Human Computer Interaction

Applying this approach means to describe this interaction as a form of rhetorical communication. To guarantee a good usability and acceptance by the user these rhetorical criteria have to be combined with established usability evaluation criteria like Norman (1988) suggested in what follows:

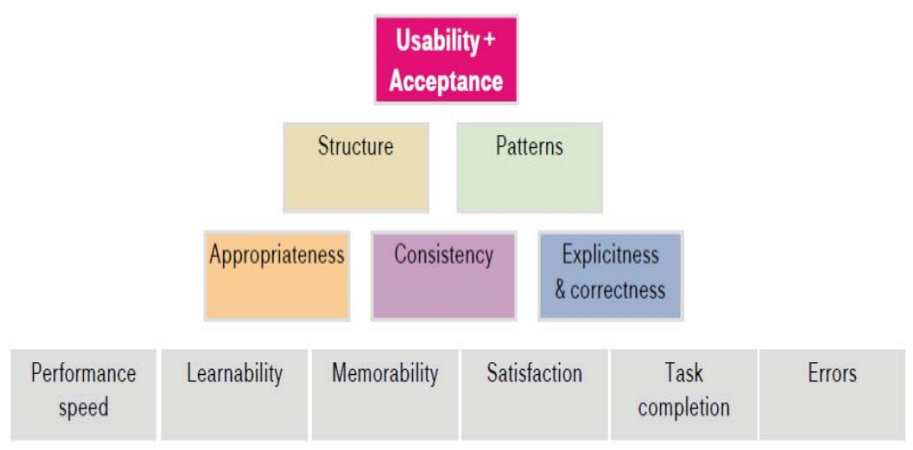

Diagram 2. Criteria for Usability \& Acceptance

This pyramid describes the criteria to plan and evaluate efficient and affective communication with system interfaces, i.e., communication and interaction between the computer and the human. Therefore, they are helpful measures for designers in the production and evaluation process. They enhance the standard usability criteria in a significant way because they focus on the appropriate addressing of the user depending on the subject, context and emotional factors.

\section{References}

Chapelle, C. 1997. 'CALL in the year 2000: Still in Search of Research Paradigms?' Language Learning \& Technology, 1(1), 19-43.

Donaldson, R. P. ve Kötter, M. 1999. 'Language Learning in Cyberspace: Teleporting the Classroom Into the Target Culture'. CALICO Journal, 16 (4): 531-558. 
Gray, R., \& Stockwell, G. (1998). 'Using Computer Mediated Communication for Language and Culture Acquisition'. On-CALL, 12(3). Retrieved July 8, 2003 from http://www.cltr.uq.edu.au/oncall/gray123.html

Hartson, H. R. (1998): Human-computer interaction: Interdisciplinary roots and trends, The Journal of Systems and Software, vol 43, pp. 103-118.

Hyland, K. 1993. 'ESL computer writers: What can we do to help?' System, 21(1), 21-29.

Kern, R. 1995. Restructuring classroom interaction with networked computers: Effects on quantity and quality of language production. Modern Language Journal, 79(4), 457-476.

Krashen, S. 1989. Language Acquisition and Language Education. New York: Prentice Hall International.

Lee, K.W. 2000. 'English Teachers' Barriers to the Use of Computer Assisted Language Learning', The Internet TESL Journal. Retrieved June, 25, 2006, from http://www.4english.cn/englishstudy/xz/thesis/barrir

Liu, M., Moore, Z., Graham, L., \& Lee, S. 2002. 'A Look at the Research on Computer-Based Technology Use in Second Language Learning: A Review of the Literature' from 1990-2000. Journal of Research on Technology in Education, 34(3), 250-273.

Norman, D. (1988), The Design of Everyday Things, Basic Books.

Singhal, M. 1998. Computer Mediated Communication (CMC): Technology for Enhancing Foreign Language/Culture Education. On-Call, 12(1). Retrieved July 8, 2003 from http://www.cltr.uq.edu.au/oncall/singhal121.html

Warschauer, M., \& Healey, D. 1998. 'Computers and Language Learning: An Overview'. Language Teaching, 31, 57-71. 